\title{
Association between contrast enhancement on contrast-enhanced CT and lenvatinib effectiveness in hepatocellular carcinoma
}

\author{
SHUSUKE OKAMURA, SHIGEO SHIMOSE, TAKASHI NIIZEKI, NAOKI KAMACHI, \\ YU NODA, TOMOTAKE SHIRONO, HIDEKI IWAMOTO, MASAHITO NAKANO, \\ RYOKO KUROMATSU, HIRONORI KOGA and TAKUJI TORIMURA \\ Division of Gastroenterology, Department of Medicine, Kurume University \\ School of Medicine, Kurume, Fukuoka 830-0011, Japan
}

Received May 10, 2021; Accepted September 19, 2021

DOI: $10.3892 / \mathrm{mco} .2021 .2442$

\begin{abstract}
Theaimof the present study was to investigate whether the degree of contrast enhancement on contrast-enhanced (CE)-CT can predict the prognosis of patients with hepatocellular carcinoma (HCC) treated with lenvatinib (LEN). A total of 67 consecutive patients with LEN-treated HCC were retrospectively analysed. In the pretreatment $\mathrm{CE}-\mathrm{CT}$, the CT values were measured using a region of interest within the main nodule and the liver parenchyma in the arterial phase, and the macroscopic degree of contrast enhancement of the tumour area was quantified by calculating the enhancement ratio (ER) of the liver parenchyma. The associations of pretreatment ER with progression-free survival (PFS) and overall survival (OS) were then investigated. There were 20,27 and 20 patients in the ER $\geq 1.5,1.0 \leq \mathrm{ER}<1.5$ and ER $<1.0$ groups, respectively. There was no significant difference in the PFS and OS among the three ER groups (PFS, $\mathrm{P}=0.63$; OS, $\mathrm{P}=0.455)$. The $\mathrm{ER}<1.0$ group had significantly more patients with larger tumour diameters, Barcelona Clinic Liver Cancer (BCLC) stage C with extrahepatic metastases, and higher des- $\gamma$-carboxy prothrombin values compared with the ER $\geq 1.0$ group, suggesting that ER $<1.0$ reflected more aggressive types of HCC. The multivariate analysis revealed tumour size and $\alpha$-fetoprotein as independent predictors of shorter PFS. Albumin-bilirubin grade 2 and BCLC stage C were significant predictors of poor OS, whereas the ER was confirmed as a non-significant predictor of both PFS and OS. Only non-alternating LEN and transarterial therapy (AT) were identified as independent predictors of unfavourable OS in patients with BCLC stage B HCC. Therefore, LEN has a
\end{abstract}

Correspondence to: Dr Shusuke Okamura, Division of Gastroenterology, Department of Medicine, Kurume University School of Medicine, 67 Asahi-machi, Kurume, Fukuoka 830-0011, Japan

E-mail: okamura_shyuusuke@kurume-u.ac.jp

Key words: hepatocellular carcinoma, lenvatinib, computed tomography, histological differentiation, prognosis strong therapeutic effect on HCC, regardless of the degree of contrast enhancement. Furthermore, AT may prolong the OS of LEN-treated patients with BCLC stage B HCC, regardless of tumour vascularity.

\section{Introduction}

Hepatocellular carcinoma (HCC) is the most common primary malignancy of the liver and is among the leading causes of cancer-related mortality worldwide (1). Most patients with HCC are diagnosed at an advanced stage, resulting in a poor prognosis (2). Recently, several types of molecular targeted agents (MTAs) have been approved for the treatment of unresectable HCC $(3,4)$. Lenvatinib (LEN) is an MTA approved as a first-line treatment; it targets vascular endothelial growth factor receptors (VEGFRs) 1-3, fibroblast growth factor receptors (FGFRs) 1-4, platelet-derived growth factor receptor- $\alpha$, RET and KIT (5). LEN exerts strong antitumour effects by inhibiting both VEGFR- and FGFR-induced angiogenesis, as well as FGFR- and RET-induced abnormal cancer cell proliferation (6).

Several studies have reported therapeutic effect-associated imaging findings of LEN (7-10). As early imaging biomarkers, a reduction in tumour enhancement intensity in the arterial phase on contrast-enhanced (CE)-CT at 2 weeks and a decrease in the time-intensity curve in the arterial phase on contrast-enhanced ultrasound at 1 week are useful predictors of LEN effectiveness $(7,8)$. On pretreatment imaging, the heterogeneous enhancement pattern of $\mathrm{HCC}$ in the arterial phase on CE-CT and ${ }^{18} \mathrm{~F}$-fluorodeoxyglucose (FDG) uptake on ${ }^{18} \mathrm{~F}-\mathrm{FDG}$-positron emission tomography (PET)/CT for HCC may be useful predictors of early response to $\operatorname{LEN}(9,10)$.

MTAs exert antitumour effects mainly by inhibiting angiogenesis. Imaging of angiogenesis is best performed using CE-CT and MRI. The enhancement pattern of HCC depends on the changes in the vasculature that occur during tumour angiogenesis (3). Clinically, however, it is unclear whether strong tumour staining is associated with a high therapeutic efficacy of MTAs, and vice versa.

To the best of our knowledge, there have been no reports to date focusing on the association between the degree of contrast enhancement on pretreatment imaging and LEN 
effectiveness in HCC. The aim of the present study was to retrospectively investigate the association between the precise degree of contrast enhancement on pretreatment CE-CT and the therapeutic efficacy of LEN in patients with HCC.

\section{Materials and methods}

Patients. Between March 2018 and December 2020, 114 consecutive patients with HCC who received LEN at Kurume University Hospital (Kurume, Japan) were enrolled in the present study. The study was conducted in accordance with the principles outlined in the Declaration of Helsinki, and the protocol was approved by the Ethics Review Committee of Kurume University (approval no. 20192). An opt-out approach was used to obtain informed consent from the patients, and personal information was protected during data collection.

The inclusion criteria for this study were as follows: i) Patients with intrahepatic tumours; ii) patients taking LEN for $>30$ days; and iii) patients who underwent CE-CT at the scheduled time prior to LEN administration. Of the 114 patients, 67 met the inclusion criteria and were subjected to further radioclinical analysis.

Administration of LEN. LEN (Eisai Co., Ltd.) was orally administered at a dose of $12 \mathrm{mg}$ for a body weight of $\geq 60 \mathrm{~kg}$ and at $8 \mathrm{mg}$ for a body weight of $<60 \mathrm{~kg}$, once per day. Adverse events (AEs) were assessed using the National Cancer Institute's Common Terminology Criteria for Adverse Events, version 4.0 (https://ctep.cancer.gov/protocoldevelopment/electronic_applications/ctc.htm). For patients with AEs of grade $\geq 3$, either LEN dose reduction or discontinuation was allowed. If required, the dose was reduced from 12 to $8 \mathrm{mg}$, or from 8 to $4 \mathrm{mg}$. In addition, alternate-day administration or 5 days-on/2 days-off (weekends-off) were also performed according to the patient's condition. The relative dose intensity (RDI) was defined as the actual dose divided by the standard dose. The 8-week RDI (8W-RDI) was calculated as the cumulative dose within the initial 8 weeks of starting LEN treatment divided by the standard dose (11).

Evaluation of therapeutic response and follow-up schedule. Therapeutic response was evaluated using dynamic CT or MRI at 4-6 weeks after the initiation of LEN according to the Modified Response Evaluation Criteria in Solid Tumours (mRECIST) (12), and at intervals of 2-3 months thereafter, until patient death or study completion.

CT protocol and image analysis. CT examinations were performed using multi-detector raw CT scanners: Discovery, GE Healthcare $(n=21)$; Revolution, GE Healthcare $(n=20)$; iCT, Philips Healthcare ( $\mathrm{n}=18)$; Aquilion, Canon Medical Systems Corporation $(\mathrm{n}=4)$; and SOMATOM, Siemens Healthineers $(\mathrm{n}=4)$. Multiphasic dynamic CT was performed with a delay of 30-40 sec (arterial phase), 60-70 sec (portal phase) and $150-180 \mathrm{sec}$ (equilibrium phase) with intravenous administration of non-ionic contrast material at a rate of 3-4 ml/sec using an automated power injector. The dose of contrast medium was adjusted according to the renal function of each patient.

If patients had multiple HCC nodules, the largest representative nodule was examined per patient. On CE-CT, the CT value was measured using a region of interest (ROI) in the arterial phase with a maximum round or oval area set within the tumour in the slice where the tumour was best visualised (almost equal to the slice including the largest diameter of the tumour) using Picture Archiving and Communication System (Fig. 1A). If the lipiodol was partially deposited in the tumour to be evaluated, the ROI was carefully defined to avoid the lipiodol deposition area based on plain (non-CE) CT (Fig. 1B). Furthermore, if the main tumour exhibited a poor contrast enhancement, we referred to ${ }^{18} \mathrm{~F}$-FDG-PET/CT to confirm FDG uptake (Fig. 1C) or previous CT or MRI scans to confirm the increase in size and to distinguish necrotic tissue from viable lesions. The CE-CT imaging protocols were uniform across all cases. However, the dose of contrast medium was reduced in some cases due to renal dysfunction. This could have influenced the contrast enhancement and, thus, the absolute $\mathrm{CT}$ value of the nodule in the arterial phase could not be used. Therefore, the background liver was set as the object. The ROI in the liver parenchyma was placed near the nodule to be evaluated in the same slice with a size of at least $100 \mathrm{~mm}^{2}$, excluding major vessels and artifacts. The enhancement ratio (ER) was calculated as follows: $\mathrm{ER}=\mathrm{CT}$ value of $\mathrm{ROI}$ in the tumour/CT value of ROI in the liver parenchyma. Furthermore, the ER values were divided into three groups: ER $<1.0$ (low contrast enhancement compared with the liver parenchyma), $1.0 \leq \mathrm{ER}<1.5$ (moderate contrast enhancement compared with the liver parenchyma) and $E R \geq 1.5$ (high contrast enhancement compared with the liver parenchyma).

Alternating LEN and transarterial therapy (AT). In addition, as will be described later, AT has been employed for cases with progressive disease (PD) at our facility. Details of AT are provided below.

AT involves the administration of treatment using a transarterial approach, such as transcatheter arterial chemoembolization (TACE) and hepatic arterial infusion chemotherapy (HAIC), upon the development of PD, as indicated by the reappearance of contrast enhancement in the tumour or appearance of new lesions in the liver during LEN treatment.

The details of AT administration were as follows: i) LEN was discontinued 2 days prior to transarterial therapy; ii) TACE was performed, except in cases exhibiting multinodular or invasive growth, in which HAIC was performed instead; iii) within 2 weeks of transarterial therapy, depending on the condition of each patient, LEN administration was resumed at the same or half the dose as that administered prior to transarterial therapy (13).

TACE and HAIC protocol. Angiography was performed for the celiac and the common hepatic arteries using a 3- or 4-Fr catheter, and digital subtraction angiography was performed using a non-ionic iodine contrast agent. The tumour-containing segment was evaluated by imaging techniques including cone-beam CT. Subsequently, a 1.7- or 1.9-Fr microcatheter (Piolax, Inc.) was inserted into the subsegmental artery with the adapted microwire (Piolax, Inc.). The catheter was advanced towards the tumour-feeding artery. Depending on the size and number of tumours, conventional TACE (C-TACE) was performed using 20-50 mg of epirubicin (Nippon Kayaku Co., Ltd.) or cisplatin (Nippon Kayaku Co., Ltd.) with lipiodol 


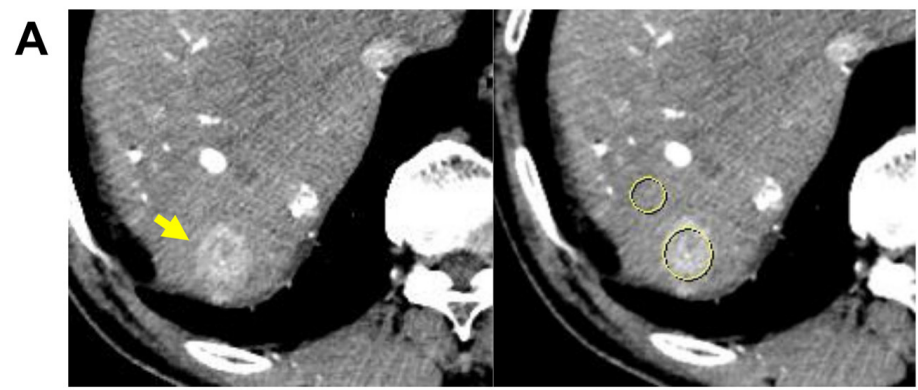

B

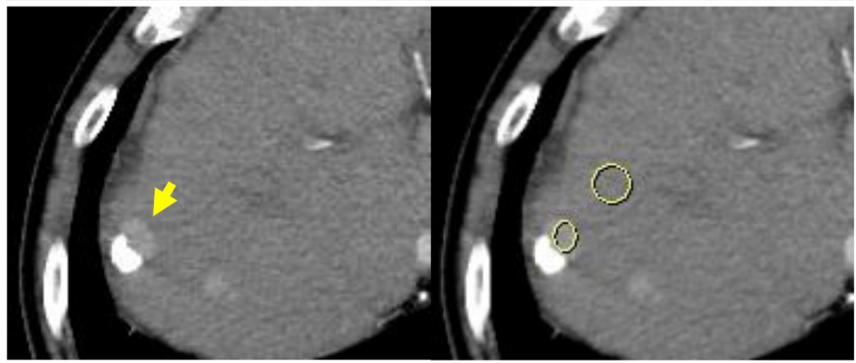

C

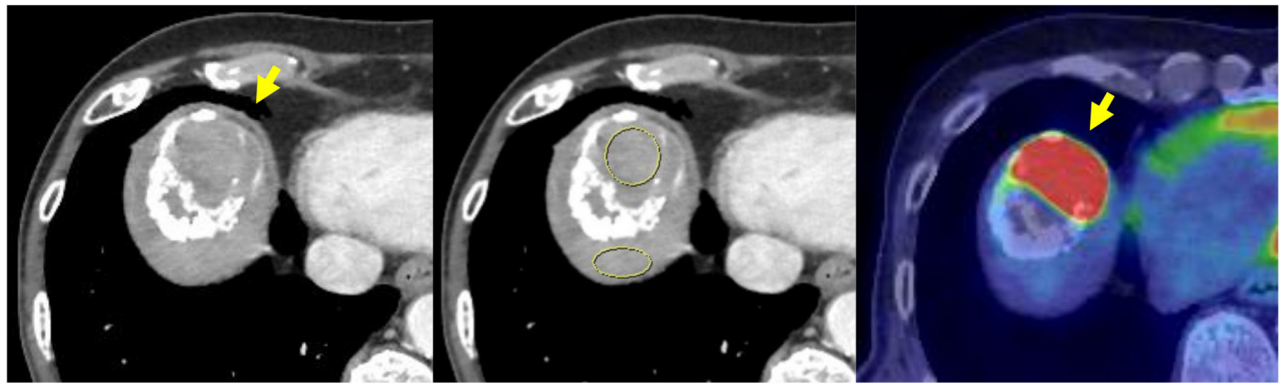

Figure 1. Method of ER measurement. (A) CT value was measured using a ROI in the arterial phase with a maximum round or oval area within the tumour in the slice where the tumour was best visualised (almost equal to the largest diameter of the tumour), and ER was calculated as follows: ER = CT value of ROI in the tumour/CT value of ROI in the liver parenchyma. (B) A case of lipiodol deposition in the main tumour. The ROI was carefully set to avoid the lipiodol deposition area. (C) A case of poor contrast enhancement in the main tumour. We referred to ${ }^{18} \mathrm{~F}-\mathrm{FDG}$ - positron emission tomography/CT to confirm FDG uptake. The ER value in the aforementioned cases was (A) 1.45 , (B) 1.38 and (C) 0.93 . Arrows are used to indicate the tumour; circles are used to indicate the ROI in the tumour and liver parenchyma. ER, enhancement ratio; ROI, region of interest; FDG, fluorodeoxyglucose.

(Guerbet Co., Ltd.), and was absorbed by gelatin sponge particles (Nippon Kayaku Co., Ltd.) (13,14).

HAIC was conducted via the insertion of an implanted catheter (Piolax, Inc.) An indwelling catheter (5-Fr W-Spiral catheter; Piolax, Inc.) was inserted through the right femoral artery, with the distal end of the catheter extending into the hepatic or gastroduodenal artery, and the proximal end connected to the port system (Soph-A-Port ${ }^{\circledR}$; Sophysa). Following the inpatient regimen of HAIC, $50 \mathrm{mg}$ of fine-powder cisplatin was suspended in 5-10 $\mathrm{ml}$ of lipiodol; the suspension volume was determined according to the tumour size [the volume (in ml) was 1-2 less than the maximum diameter (in $\mathrm{cm})$ ]. On day 1 , the cisplatin-lipiodol suspension was injected through the implanted angiography catheter, followed by the injection of $250 \mathrm{mg}$ of 5-fluorouracil (5-FU). Then, 1,250 mg of 5-FU was continuously infused for 5 days using an infusion balloon pump (Surefuser ${ }^{\mathrm{TM}}+$, Nipro Pharma Corporation). This regimen was administered once per week during the first 2 weeks of admission, and then a combination of $20 \mathrm{mg}$ cisplatin with lipiodol and 5-FU (500-1,250 mg) was infused every 2 weeks at the outpatient department until disease progression $(13,15)$.

Statistical analysis. Continuous variables are expressed as median (range). The $\chi^{2}$ test or Fisher's exact test were used to analyse the association between categorical variables, and Wilcoxon's test was used to analyse the association between continuous variables. Progression-free survival (PFS) and overall survival (OS) were calculated from the date of initiation of LEN administration to tumour progression and death, respectively. A Cox proportional hazard model was used for univariate and multivariate analyses to identify any independent variables associated with PFS and OS. The PFS and OS rates were evaluated using the Kaplan-Meier method, and the log-rank test was used to compare the patient groups. $\mathrm{P}<0.05$ was considered to indicate statistically significant differences. JMP software (version 15; SAS Institute, Inc.) was used for all statistical analyses.

\section{Results}

Patient and tumour characteristics. The patient and tumour characteristics are summarised in Table I. The majority of the patients had Child-Pugh class A liver cirrhosis, with the exception of 2 patients with class B cirrhosis. Albumin-bilirubin (ALBI) grade 1 and 2 were observed in 30 and 37 patients, respectively. A total of 2 patients had stage A tumours, 43 had stage $B$ tumours and 22 had stage $C$ tumours as per the Barcelona Clinic Liver Cancer (BCLC) staging system (16). Only 1 patient had macrovascular invasion; 21 of the 22 patients 
Table I. Patient and tumour characteristics.

\begin{tabular}{lc}
\hline Characteristics & $\mathrm{n}=67$ \\
\hline Age (years) & $73(47-90)$ \\
Sex (male/female) & $54 / 13$ \\
Aetiology (HBV/HCV/others) & $9 / 30 / 28$ \\
Albumin (g/dl) & $3.9(2.9-4.7)$ \\
Total bilirubin (mg/dl) & $0.7(0.4-1.9)$ \\
Prothrombin activity (\%) & $98(56-130)$ \\
Child-Pugh score (A/B/C) & $65 / 2 / 0$ \\
ALBI grade $(1 / 2 / 3)$ & $30 / 37 / 0$ \\
Tumour number $(<5 / 5-10 / \geq 10)$ & $30 / 18 / 19$ \\
Tumour size (mm) & $25(10-170)$ \\
Macrovascular invasion (present/absent) & $1 / 66$ \\
Extrahepatic metastasis (present/absent) & $21 / 46$ \\
BCLC stage $(\mathrm{A} / \mathrm{B} / \mathrm{C})$ & $2 / 43 / 22$ \\
Up-to-seven criteria (within/beyond) & $25 / 42$ \\
AFP (ng/ml) & $25.5(1.4-118,560)$ \\
DCP (mAU/ml) & $396(12-179,531)$ \\
TACE refractoriness (yes/no) & $51 / 16$ \\
ER & $1.28(0.01-2.30)$ \\
ER <1 $.0 / 1.0 \leq$ ER <1.5/ER $\geq 1.5$ & $20 / 27 / 20$ \\
Initial dose of lenvatinib (8/12 mg) & $46 / 21$ \\
$8 W-R D I ~(\%)$ & $75(15-100)$ \\
\hline
\end{tabular}

Continuous variables are expressed as median (range). HBV, hepatitis B virus; $\mathrm{HCV}$, hepatitis $\mathrm{C}$ virus; $\mathrm{ALBI}$ grade, albumin-bilirubin grade; BCLC stage, Barcelona Clinic Liver Cancer stage; AFP, $\alpha$-foetoprotein; DCP, des- $\gamma$-carboxy prothrombin; TACE, transcatheter arterial chemoembolization; ER, enhancement ratio; 8W-RDI, relative dose intensity after 8 weeks of lenvatinib induction.

with BCLC stage C HCC had extrahepatic metastasis. There were 25 patients within up-to-seven criteria [defined as HCC with 7 as the sum of the size of the largest tumour (in $\mathrm{cm}$ ) and the number of intrahepatic tumours] and 42 beyond this criteria.

Among the 67 patients, 51 had HCC that was refractory to TACE. C-TACE was performed in 46 patients and drug-eluting beads TACE (DEB-TACE) was performed in 5 patients as pretreatment of LEN. Lesions treated with DEB-TACE may display reduced enhancement and lower ER. However, since the main nodules to be evaluated in these cases were new lesions appearing after DEB-TACE $(n=1)$, or previously existing lesions increasing in size with no therapeutic efficacy $(n=2)$, or lesions displaying tumour angiogenesis resumption and increase in size after DEB-TACE $(n=2)$, it was hypothesized that DEB-TACE did not affect the evaluation of the contrast enhancement in this study. Therefore, these cases were included in the present analysis. The initial dose of LEN was $8 \mathrm{mg}$ for 46 patients and $12 \mathrm{mg}$ for 21 patients. The RDI at 8 weeks was $75 \%$ (range, 15-100\%). By the time LEN was discontinued, dose reduction had been performed in $50(74.6 \%)$ patients. The main reasons for dose reduction were fatigue $(n=14)$, decreased appetite $(n=10)$, proteinuria $(n=4)$, ascites $(n=4)$, palmar-plantar erythrodysaesthesia syndrome $(n=3)$, diarrhoea $(n=3)$, elevated aspartate aminotransferase levels $(n=2)$, high ammonia levels $(n=2)$, hypertension $(n=1)$, hoarseness $(n=1)$, neutropenia $(n=1)$, increased blood bilirubin levels $(n=1)$, hyperthyroidism $(n=1)$, vomiting $(\mathrm{n}=1)$, headache $(\mathrm{n}=1)$ and gingival bleeding $(\mathrm{n}=1)$. The contrast medium dose was reduced in 24 patients (35.8\%) due to renal dysfunction. The CT values in the ROI of the tumour and liver parenchyma were 100.56 Hounsfield units (HU) (range, 0.48-169.86 HU) and 80.79 HU (range, 54.79-113.41 HU), respectively. The areas of the ROI in the tumour and liver parenchyma were $176.98 \mathrm{~mm}^{2}$ (range, 25.51-5332.46 $\mathrm{mm}^{2}$ ) and $256.68 \mathrm{~mm}^{2}$ (range, $112.55-947.37 \mathrm{~mm}^{2}$ ), respectively. The median ER was 1.28 (range, 0.01-2.3).

Evaluation using mRECIST after the initial treatment with $L E N$. Complete response, partial response, stable disease and PD were observed in $10.4 \%$ (7/67), 46.3\% (31/67), $32.8 \%(22 / 67)$ and $10.4 \%$ (7/67) of the patients, respectively. The overall objective response rate (ORR) and disease control rate (DCR) were 56.7\% (38/67) and 89.6\% (60/67), respectively.

Comparison of PFS and OS among the ER groups. The PFS and OS curves stratified by ER in the arterial phase on CE-CT are shown in Fig. 2. The ER $\geq 1.5,1.0 \leq \mathrm{ER}<1.5$ and $\mathrm{ER}<1.0$ groups comprised 20, 27 and 20 patients, respectively. There was no significant difference among the ER groups (PFS, $\mathrm{P}=0.63$; OS, $\mathrm{P}=0.455$ ).

Comparison of patient and tumour characteristics between the $E R \geq 1.0$ and $<1.0$ groups. Next, we focused on comparing baseline patient and tumour characteristics between the ER $\geq 1.0$ and ER $<1.0$ groups, based on the high or low contrast enhancement compared with that of the liver parenchyma. A comparison of patient and tumour characteristics between the two groups is shown in Table II. The ER $<1.0$ group comprised significantly more cases with larger tumour diameters $(\mathrm{P}<0.001)$, BCLC stage $\mathrm{C}$ with extrahepatic metastases $(\mathrm{P}=0.007)$ and higher des- $\gamma$-carboxy prothrombin (DCP) values $(\mathrm{P}=0.046)$ compared with the $\mathrm{ER} \geq 1.0$ group, suggesting that ER $<1.0$ was characteristic of aggressive types of HCC. There was no significant difference in the ORR and $\mathrm{DCR}$ between the ER groups (ORR, $\mathrm{P}=0.072$; $\mathrm{DCR}, \mathrm{P}=0.938$ ).

Factors associated with PFS and OS. Independent predictors of PFS (Table III) and OS (Table IV) were investigated using Cox proportional hazard analysis in all patients. The results of the univariate analysis showed that tumour size $(\geq 30 \mathrm{~mm}$; $\mathrm{P}=0.02), \alpha$-foetoprotein (AFP) ( $\geq 100 \mathrm{ng} / \mathrm{ml} ; \mathrm{P}=0.04)$ and DCP ( $\geq 200 \mathrm{mAU} / \mathrm{ml} ; \mathrm{P}=0.03$ ) were significant risk factors for PFS. The multivariate analysis revealed that tumour size $(\geq 30 \mathrm{~mm}$; $\mathrm{HR}=2.14 ; 95 \% \mathrm{CI}: 1.18-3.85 ; \mathrm{P}=0.012)$ and AFP $(\geq 100 \mathrm{ng} / \mathrm{ml}$; $\mathrm{HR}=1.98 ; 95 \% \mathrm{CI}: 1.08-3.63 ; \mathrm{P}=0.026)$ were independent predictors of shorter PFS. The results of the univariate analysis showed that $\mathrm{BCLC}$ stage $\mathrm{C}(\mathrm{P}=0.019)$ was a significant risk factor for OS. The multivariate analysis revealed that ALBI grade $2(\mathrm{HR}=2.60 ; 95 \% \mathrm{CI}: 1.08-6.26 ; \mathrm{P}=0.034)$ and $\mathrm{BCLC}$ stage $\mathrm{C}(\mathrm{HR}=2.86 ; 95 \% \mathrm{CI}: 1.28-6.38 ; \mathrm{P}=0.011)$ were independent predictors of poor OS. The ER was confirmed to be a non-significant predictor of both PFS and OS.

Factors associated with $O S$ in patients with BCLC stage B $H C C$. As there were $43 \mathrm{HCC}$ patients with BCLC B, among 
Table II. Comparisons of patient and tumour characteristics based on the ER.

\begin{tabular}{|c|c|c|c|}
\hline Characteristics & $\mathrm{ER} \geq 1.0$ & $\mathrm{ER}<1.0$ & P-value \\
\hline Age (years) & $76(54-90)$ & $73(47-88)$ & 0.344 \\
\hline Sex (male/female) & $37 / 10$ & $16 / 4$ & 0.906 \\
\hline Aetiology (HBV/HCV/others) & $5 / 23 / 19$ & $4 / 7 / 9$ & 0.451 \\
\hline Albumin $(\mathrm{g} / \mathrm{dl})$ & $3.8(2.9-4.7)$ & $4.0(3.1-4.4)$ & 0.287 \\
\hline Total bilirubin (mg/dl) & $0.73(0.4-1.9)$ & $0.70(0.5-1.3)$ & 0.967 \\
\hline Prothrombin activity (\%) & $96(56-124)$ & $103(76-130)$ & 0.415 \\
\hline Child-Pugh score (A/B/C) & $45 / 2 / 0$ & $20 / 0 / 0$ & 0.349 \\
\hline ALBI grade $(1 / 2 / 3)$ & $18 / 29 / 0$ & $12 / 8 / 0$ & 0.102 \\
\hline Tumour number $(<5 / 5-10 / \geq 10)$ & $18 / 15 / 14$ & $12 / 3 / 5$ & 0.214 \\
\hline Tumour size (mm) & $22(10-170)$ & $42(17-122)$ & $<0.001$ \\
\hline Macrovascular invasion (present/absent) & $1 / 46$ & $0 / 20$ & 0.511 \\
\hline Extrahepatic metastasis (present/absent) & $9 / 38$ & $12 / 8$ & 0.001 \\
\hline BCLC stage (A/B/C) & $2 / 35 / 10$ & $0 / 8 / 12$ & 0.007 \\
\hline Up-to-seven criteria (within/beyond) & $17 / 30$ & $8 / 12$ & 0.767 \\
\hline AFP (ng/ml) & $26.1(1.6-40804)$ & $25.0(1.4-118560)$ & 0.827 \\
\hline $\mathrm{DCP}(\mathrm{mAU} / \mathrm{ml})$ & $232(12-15120)$ & $1408(22-179531)$ & 0.046 \\
\hline TACE refractoriness (yes/no) & $40 / 7$ & $11 / 9$ & 0.008 \\
\hline ER & $1.45(1.01-2.30)$ & $0.84(0.01-0.98)$ & $<0.001$ \\
\hline Initial dose of lenvatinib $(8 / 12 \mathrm{mg})$ & $34 / 13$ & $12 / 8$ & 0.319 \\
\hline $8 \mathrm{~W}-\mathrm{RDI}(\geq 75 \% /<75 \%)$ & $24 / 23$ & $10 / 10$ & 0.937 \\
\hline Objective response rate & $30(63.8 \%)$ & $8(40 \%)$ & 0.072 \\
\hline Disease control rate & $42(89.3 \%)$ & $18(90 \%)$ & 0.938 \\
\hline
\end{tabular}

Continuous variables are expressed as median (range). HBV, hepatitis B virus; HCV, hepatitis C virus; ALBI grade, albumin-bilirubin grade; BCLC stage, Barcelona Clinic Liver Cancer stage; AFP, $\alpha$-foetoprotein; DCP, des- $\gamma$-carboxy prothrombin; TACE, transcatheter arterial chemoembolization; ER, enhancement ratio; 8W-RDI, relative dose intensity after 8 weeks of lenvatinib induction.
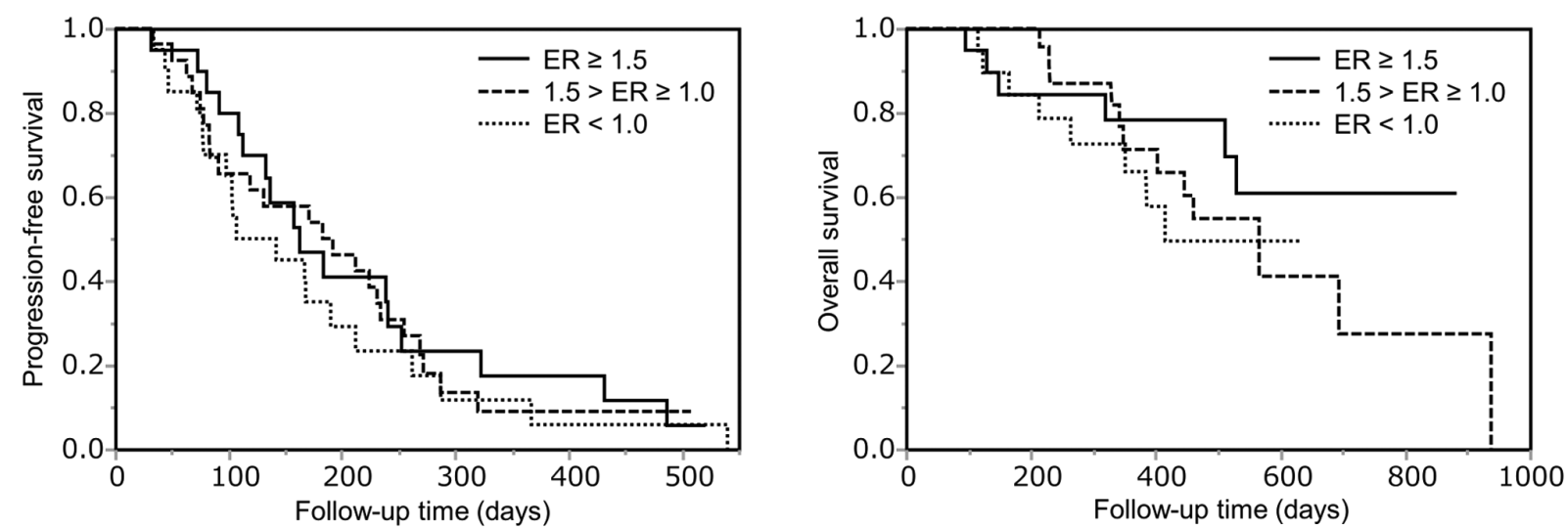

Figure 2. Kaplan-Meier curves of the estimated PFS and OS based on the differences in the ER. The groups with ER $\geq 1.5$, $1.0 \leq \mathrm{ER}<1.5$ and ER $<1.0$ included 20, 27 and 20 patients, respectively. There was no significant difference among the ER groups $(P F S, P=0.63$; OS, $P=0.455)$. ER, enhancement ratio; PFS, progression-free survival; OS, overall survival.

whom 23 patients $(53 \%)$ underwent AT as a post-PD treatment, it was considered important that the effect of such additive AT on OS was assessed. Therefore, the OS of 43 patients with BCLC stage B was analysed, focusing on the presence or absence of AT (Table V). The univariate analysis revealed that ALBI grade $2(\mathrm{HR}=4.77 ; 95 \% \mathrm{CI}: 1.05-21.55 ; \mathrm{P}=0.043)$ and non-AT $(\mathrm{HR}=20.95$; 95\% CI: 2.7-162.55; $\mathrm{P}=0.004)$ were significant factors affecting OS. The multivariate analysis revealed only non-AT as an independent predictor of unfavourable OS (HR=16.42; 95\% CI: 2.03-133.04; $\mathrm{P}=0.009)$. ER was not identified as a significant predictor in this analysis.

\section{Discussion}

The present study evaluated the association between the precise degree of contrast enhancement and the therapeutic 
Table III. Univariate and multivariate analyses of progression-free survival of patients with hepatocellular carcinoma.

\begin{tabular}{|c|c|c|c|c|}
\hline \multirow[b]{2}{*}{ Variables } & \multicolumn{2}{|c|}{ Univariate analysis } & \multicolumn{2}{|c|}{ Multivariate analysis } \\
\hline & $\mathrm{HR}(95 \% \mathrm{Cl})$ & P-value & $\mathrm{HR}(95 \% \mathrm{Cl})$ & P-value \\
\hline Age ( $\geq 70$ years $)$ & $0.96(0.56-1.66)$ & 0.883 & & \\
\hline Sex (male) & $0.97(0.50-1.87)$ & 0.916 & & \\
\hline HCV positivity & $1.62(0.95-2.78)$ & 0.073 & $1.60(0.93-2.77)$ & 0.091 \\
\hline ALBI grade 2 & $1.17(0.69-1.96)$ & 0.561 & & \\
\hline Tumour number $(\geq 5)$ & $1.04(0.62-1.75)$ & 0.882 & & \\
\hline Tumour size ( $\geq 30 \mathrm{~mm})$ & $1.85(1.10-3.12)$ & 0.020 & $2.14(1.18-3.85)$ & 0.012 \\
\hline BCLC stage C & $1.12(0.64-1.96)$ & 0.679 & & \\
\hline Up-to-seven criteria (beyond) & $1.36(0.79-2.33)$ & 0.272 & & \\
\hline $\operatorname{AFP}(\geq 100 \mathrm{ng} / \mathrm{ml})$ & $1.76(1.03-3.00)$ & 0.040 & $1.98(1.08-3.63)$ & 0.026 \\
\hline $\mathrm{DCP}(\geq 200 \mathrm{mAU} / \mathrm{ml})$ & $1.82(1.06-3.12)$ & 0.030 & $1.33(0.75-2.36)$ & 0.329 \\
\hline TACE refractoriness & $1.06(0.58-1.95)$ & 0.839 & & \\
\hline $\mathrm{ER}<1.0$ & $1.30(0.74-2.26)$ & 0.364 & & \\
\hline Initial dose of lenvatinib $8 \mathrm{mg}$ & $1.55(0.89-2.72)$ & 0.123 & & \\
\hline $8 \mathrm{~W}-\mathrm{RDI}(<75 \%)$ & $1.53(0.91-2.58)$ & 0.108 & & \\
\hline
\end{tabular}

Continuous variables are expressed as median (range). HCV, hepatitis C virus; ALBI grade, albumin-bilirubin grade; BCLC stage, Barcelona Clinic Liver Cancer stage; AFP, $\alpha$-foetoprotein; DCP, des- $\gamma$-carboxy prothrombin; TACE, transcatheter arterial chemoembolization; ER, enhancement ratio; 8W-RDI, relative dose intensity after 8 weeks of lenvatinib induction.

Table IV. Univariate and multivariate analyses of overall survival of patients with hepatocellular carcinoma.

\begin{tabular}{|c|c|c|c|c|}
\hline \multirow[b]{2}{*}{ Variables } & \multicolumn{2}{|c|}{ Univariate analysis } & \multicolumn{2}{|c|}{ Multivariate analysis } \\
\hline & $\mathrm{HR}(95 \% \mathrm{Cl})$ & P-value & $\mathrm{HR}(95 \% \mathrm{Cl})$ & P-value \\
\hline Age ( $\geq 70$ years $)$ & $0.98(0.42-2.28)$ & 0.966 & & \\
\hline Sex (male) & $1.06(0.40-2.84)$ & 0.908 & & \\
\hline HCV positivity & $1.15(0.52-2.52)$ & 0.729 & & \\
\hline ALBI grade 2 & $2.34(0.98-5.61)$ & 0.057 & $2.60(1.08-6.26)$ & 0.034 \\
\hline Tumour number $(\geq 5)$ & $1.06(0.48-2.37)$ & 0.884 & & \\
\hline Tumour size ( $\geq 30 \mathrm{~mm})$ & $1.71(0.78-3.77)$ & 0.181 & & \\
\hline BCLC stage C & $2.58(1.17-5.69)$ & 0.019 & $2.86(1.28-6.38)$ & 0.011 \\
\hline Up-to-seven criteria (beyond) & $1.18(0.51-2.75)$ & 0.694 & & \\
\hline $\operatorname{AFP}(\geq 100 \mathrm{ng} / \mathrm{ml})$ & $1.70(0.77-3.73)$ & 0.186 & & \\
\hline DCP ( $\geq 200 \mathrm{mAU} / \mathrm{ml})$ & $1.82(0.79-4.19)$ & 0.157 & & \\
\hline TACE refractoriness & $1.09(0.41-2.94)$ & 0.859 & & \\
\hline $\mathrm{ER}<1.0$ & $1.47(0.63-3.46)$ & 0.373 & & \\
\hline
\end{tabular}

Continuous variables are expressed as median (range). HCV, hepatitis C virus; ALBI grade, albumin-bilirubin grade; BCLC stage, Barcelona Clinic Liver Cancer stage; AFP, $\alpha$-foetoprotein; DCP, des- $\gamma$-carboxy prothrombin; TACE, transcatheter arterial chemoembolization; ER, enhancement ratio.

efficacy of LEN. Through various analyses, it was observed that there was no significant difference in PFS and OS among the ER groups; therefore, ER was not found to be a significant predictor in LEN-treated patients with HCC.

On dynamic CT, hypoattenuation in the arterial phase is frequently observed in both well-differentiated and poorly differentiated HCCs $(17,18)$. Furthermore, sarcomatous hepatic tumours generally exhibit hypovascularity, which is seen as rim enhancement or non-enhancement on arterial phase imaging (19). Thus, nodules with poor contrast enhancement in the arterial phase are considered as either less or highly malignant. The present study included two cases of BCLC stage A (both in the ER >1.5 group); however, advanced or unresectable HCCs, for which LEN treatment is usually recommended, are in BCLC stage B or C (2). Although this could not be ascertained in the present study, as histopathological 
Table V. Univariate and multivariate analyses of overall survival of patients with BCLC stage B hepatocellular carcinoma ( $\mathrm{n}=43$ ).

\begin{tabular}{|c|c|c|c|c|}
\hline \multirow[b]{2}{*}{ Variables } & \multicolumn{2}{|c|}{ Univariate analysis } & \multicolumn{2}{|c|}{ Multivariate analysis } \\
\hline & $\mathrm{HR}(95 \% \mathrm{Cl})$ & $\mathrm{P}$-value & $\mathrm{HR}(95 \% \mathrm{Cl})$ & $\mathrm{P}$-value \\
\hline Age ( $\geq 70$ years) & $1.33(0.36-4.86)$ & 0.670 & & \\
\hline Sex (male) & $0.97(0.27-3.53)$ & 0.963 & & \\
\hline HCV positivity & $0.85(0.28-2.53)$ & 0.769 & & \\
\hline ALBI grade 2 & $4.77(1.05-21.55)$ & 0.043 & $2.12(0.45-9.99)$ & 0.341 \\
\hline Tumour number $(\geq 5)$ & $1.06(0.33-3.53)$ & 0.892 & & \\
\hline Tumour size ( $\geq 30 \mathrm{~mm})$ & $1.08(0.38-3.66)$ & 0.770 & & \\
\hline Up-to-seven criteria (beyond) & $0.96(0.29-3.11)$ & 0.943 & & \\
\hline $\operatorname{AFP}(\geq 100 \mathrm{ng} / \mathrm{ml})$ & $1.37(0.46-4.07)$ & 0.576 & & \\
\hline $\mathrm{DCP}(\geq 200 \mathrm{mAU} / \mathrm{ml})$ & $2.16(0.69-6.72)$ & 0.180 & & \\
\hline $\mathrm{ER}<1.0$ & $0.91(0.20-4.11)$ & 0.901 & & \\
\hline Non-AT & $20.95(2.7-162.55)$ & 0.004 & $16.42(2.03-133.04)$ & 0.009 \\
\hline
\end{tabular}

examinations were not performed immediately prior to LEN administration, it is unlikely that well-differentiated HCCs were included. In fact, in the present study, the number of patients with aggressive HCC, characterized by large size, extrahepatic metastases and high DCP values, was significantly higher in the ER $<1.0$ group compared with the other groups. Although such HCCs are refractory to various treatments and have a poor prognosis $(20,21)$, no significant difference in PFS has been reported. Previously, Kawamura et al (9) examined the association between PFS and the pretreatment enhancement patterns of HCC on CE-CT in LEN-treated patients. The patterns were defined as homogeneous or heterogeneous, and it was concluded that there was no difference in PFS between the patterns, despite the heterogeneity indicating the highly malignant nature of $\operatorname{HCC}(9,22)$. Although that evaluation method was quite different from the one used in the present study, the findings suggest that LEN exerts a strong therapeutic effect regardless of the degree of HCC differentiation.

In human HCC, CTNNB1 and TP53 mutations define two distinct tumour phenotypes, and histological subtypes are associated with clinical and molecular characteristics (23). The CTNNB1 mutation is frequent, even in well-differentiated HCC, whereas TP53 mutations are more frequent in poorly differentiated HCCs and those with foci of sarcomatous change $(23,24)$. Recently, Rodríguez-Hernández et al (25) reported that LEN was more effective in moderately-to-poorly differentiated liver cancer cells with the p53 mutation. Thus, these reports support our finding that LEN is effective against moderately-to-poorly differentiated HCC.

In the present study, the ER was not identified as a significant predictor of PFS or OS. The multivariate analyses demonstrated that ALBI grade 2 and BCLC stage $C$ were independent predictors of poor OS. ALBI grade is useful in predicting prognosis after HCC treatment, such as liver resection, radiofrequency ablation and TACE (26-28). Regarding the predictive potential of the ALBI grade in LEN treatment,
Ueshima et al (29) reported that HCC patients with ALBI grade 1 presented a higher response rate and lower frequency of treatment discontinuation due to severe AEs. Furthermore, Hiraoka et al (30) reported that modified ALBI grade 2 b or 3 was superior to Child-Pugh classification in predicting poor prognosis of patients with HCC receiving LEN treatment. Although the modified ALBI grading system was not used in the present study, classical ALBI grade 2 was found to be a prognostic factor in patients with $\mathrm{HCC}$ receiving LEN treatment.

As there were 23 patients (53\%) with BCLC stage B HCC in whom AT was performed after PD, a sub-analysis of OS in BCLC stage B patients was performed. Although 38 of the 43 patients $(88 \%)$ treated with LEN had TACE-refractory $\mathrm{HCC}$, the additive effect of AT significantly prolonged OS in this study. Previously, Kudo et al (31) reported that TACE plus sorafenib significantly improved PFS compared with TACE alone in patients with unresectable HCC. They considered that sorafenib-induced normalisation of tumour blood vessels leads to increased accumulation of TACE-derived mixture of lipiodol and anticancer agent compared with TACE alone. LEN is also an angiogenesis inhibitor, similar to sorafenib; therefore, it was suggested that a similar mechanism was involved in the limited number of patients with BCLC stage B disease. Accordingly, normalisation of the tumour vasculature may increase responsiveness to AT in hypovascular HCCs, which are known to hardly respond to TACE $(32,33)$.

Recently, Shimose et al (13) reported that AT prolonged OS in LEN-treated patients with BCLC stage B HCC in the propensity score matching (PSM) analysis of their multicentre study. They deduced the mechanism underlying the efficacy of AT as follows: Transarterial therapy-induced intratumour ischaemia upregulates hypoxia-inducible factor 1- $\alpha$ expression, which leads to increased expression of its downstream angiogenic factors for tumour growth, and LEN administration after transarterial therapy suppresses the effects of these 
angiogenic factors $(34,35)$. Similarly, it was considered reasonable that the AT strategy contributed to the improved OS of the LEN-treated patients with BCLC stage B HCC in the present study.

There were certain limitations to the present study. First, this was a retrospective study that involved a small number of patients from a single centre; thus, there was a possibility of selection bias. Furthermore, the ER $<1.0$ group with larger tumour diameters, BCLC stage $\mathrm{C}$ with extrahepatic metastases and higher DCP values, consisted of only 20 patients, which may represent possible confounding factors and lead to underestimation of the impact of ER on PFS and OS. This may be resolved by matching tumour factors with PSM analysis in a larger number scale. Second, only one case of macrovascular invasion was enrolled. Thus, PFS and OS could not be analysed in HCC patients with macrovascular invasion. Third, a histological examination was not performed immediately prior to LEN administration. Therefore, the result of the present study was only the association between the contrast enhancement and the therapeutic effect. However, procedures like aspiration biopsy are invasive and have drawbacks, including sampling errors. Therefore, histological examination is often not performed immediately prior to LEN administration in the clinical setting. Therefore, we believe that this result may have potential benefits for LEN treatment. Finally, the ER used in the present study may be affected by the haemodynamic condition of organs, the types of CT devices and the types of contrast media, regardless of their superiority over absolute CT values in assessing the contrast enhancement on the tumour. Further studies are needed to determine whether pretreatment degree of contrast enhancement is important through prospective analyses in a larger number of cases with a unified CT device.

In conclusion, the lower pretreatment contrast enhancement in HCC did not contribute to the prediction of the PFS or OS in patients with LEN-treated HCC. The results of the present study suggested that LEN exerted a strong therapeutic effect on HCC, regardless of the degree of contrast enhancement in the tumour. In addition, AT may prolong the OS of patients with LEN-treated BCLC stage B HCC, regardless of the tumour vascularity.

\section{Acknowledgements}

Not applicable.

\section{Funding}

No funding was received.

\section{Availability of data and materials}

The datasets used and/or analysed during the current study are available from the corresponding author on reasonable request.

\section{Authors' contributions}

SO participated in the conception and design of study, acquisition and interpretation of data, and drafting of the manuscript. SS participated in the conception and design of the study, acquisition and interpretation of data. SO and SS have seen and can confirm the authenticity of the raw data. TN, NK, YN, TS, HI, MN and RK participated in the acquisition of data. HK and TT participated in the interpretation of data and critical revision of the manuscript for important intellectual content. All the authors have read and approved the final manuscript.

\section{Ethics approval and consent to participate}

The present study was conducted in accordance with the Declaration of Helsinki, and the protocol was approved by the Ethics Review Committee of Kurume University (approval no. 20192). An opt-out approach was used to obtain informed consent from the patients, and personal information was protected during data collection.

\section{Patient consent for publication}

Not applicable.

\section{Competing interests}

The authors declare that they have no competing interests.

\section{References}

1. Bray F, Ferlay J, Soerjomataram I, Siegel RL, Torre LA and Jemal A: Global cancer statistics 2018: GLOBOCAN estimates of incidence and mortality worldwide for 36 cancers in 185 countries. CA Cancer J Clin 68: 394-424, 2018.

2. Galle PR, Forner A, Llovet JM, Mazzaferro V, Piscaglia F, Raoul JL, Schirmacher P and Vilgrain V; European Association for the Study of the Liver. Electronic address: easloffice@easloffice.eu; European Association for the Study of the Liver: EASL Clinical Practice Guidelines: Management of hepatocellular carcinoma. J Hepatol 69: 182-236, 2018.

3. Moawad AW, Szklaruk J, Lall C, Blair KJ, Kaseb AO, Kamath A, Rohren SA and Elsayes KM: Angiogenesis in hepatocellular carcinoma; pathophysiology, targeted therapy, and role of imaging. J Hepatocell Carcinoma 7: 77-89, 2020.

4. Heimbach JK, Kulik LM, Finn RS, Sirlin CB, Abecassis MM, Roberts LR, Zhu AX, Murad MH and Marrero JA: AASLD guidelines for the treatment of hepatocellular carcinoma. Hepatology 67: 358-380, 2018.

5. Kudo M, Finn RS, Qin S, Han KH, Ikeda K, Piscaglia F, Baron A, Park JW, Han G, Jassem J, et al: Lenvatinib versus sorafenib in first-line treatment of patients with unresectable hepatocellular carcinoma: A randomised phase 3 non-inferiority trial. Lancet 391: 1163-1173, 2018.

6. Yamamoto Y, Matsui J, Matsushima T, Obaishi H, Miyazaki K, Nakamura K, Tohyama O, Semba T, Yamaguchi A, Hoshi SS, et al: Lenvatinib, an angiogenesis inhibitor targeting VEGFR/FGFR, shows broad antitumor activity in human tumor xenograft models associated with microvessel density and pericyte coverage. Vasc Cell 6: 18, 2014.

7. Kunimoto H, Shakado S, Tanaka T, Takata K, Yamauchi R, Fukuda H, Tsuchiya N, Yokoyama K, MoriharaD, Takeyama Y, et al: Reduction in tumor stain at 2 weeks after treatment initiation is a predictor of the efficacy of lenvatinib in patients with unresectable hepatocellular carcinoma. Oncology 98: 779-786, 2020.

8. Kuorda H, Abe T, Fujiwara Y, Okamoto T, Yonezawa M, Sato H, Endo K, Oikawa T, Sawara K and Takikawa Y: Change in arterial tumor perfusion is an early biomarker of lenvatinib efficacy in patients with unresectable hepatocellular carcinoma. World J Gastroenterol 25: 2365-2372, 2019.

9. Kawamura Y, Kobayashi M, Shindoh J, Kobayashi Y, Kasuya K, Sano T, Fujiyama S, Hosaka T, Saitoh S, Sezaki H, et al: Pretreatment heterogeneous enhancement pattern of hepatocellular carcinoma may be a useful new predictor of early response to lenvatinib and overall prognosis. Liver Cancer 9: $275-292,2020$ 
10. Kawamura Y, Kobayashi M, Shindoh J, Kobayashi Y, Kasuya K, Sano T, Fujiyama S, Hosaka T, Saitoh S, Sezaki H, et al: ${ }^{18}$ F-fluorodeoxyglucose uptake in hepatocellular carcinoma as a useful predictor of an extremely rapid response to lenvatinib. Liver Cancer 9: 84-92, 2020.

11. Takahashi A, Moriguchi M, Seko Y, Ishikawa H, Yo T, Kimura H, Fujii $\mathrm{H}$, Shima T, Mitsumoto Y, Ishiba $\mathrm{H}$, et al: Impact of relative dose intensity of early-phase lenvatinib treatment on therapeutic response in hepatocellular carcinoma. Anticancer Res 39: 5149-5156, 2019.

12. Lencioni R and Llovet JM: Modified RECIST (mRECIST) assessment for hepatocellular carcinoma. Semin Liver Dis 30: $52-60,2010$.

13. Shimose S, Iwamoto H, Tanaka M, Niizeki T, Shirono T, Noda Y, Kamachi N, Okamura S, Nakano M, Suga H, et al: Alternating lenvatinib and trans-arterial therapy prolongs overall survival in patients with inter-mediate stage hepatocellular carcinoma: A propensity score matching study. Cancers (Basel) 13: 160, 2021.

14. Shimose S, Kawaguchi T, Iwamoto H, Niizeki T, Shirono T, Tanaka M, Koga $\mathrm{H}$ and Torimura T: Indication of suitable transarterial chemoembolization and multikinase inhibitors for intermediate stage hepatocellular carcinoma. Oncol Lett 19: 2667-2676, 2020.

15. Nagamatsu $H$, Sumie $S$, Niizeki T, Tajiri N, Iwamoto $H$, Aino $H$, Nakano M, Shimose S, Satani M, Okamura S, et al: Hepatic arterial infusion chemoembolization therapy for advanced hepatocellular carcinoma: Multicenter phase II study. Cancer Chemother Pharmacol 77: 243-250, 2016.

16. Llovet JM, Di Bisceglie AM, Bruix J, Kramer BS, Lencioni R, Zhu AX, Sherman M, Schwartz M, Lotze M, Talwalkar J, et al; Panel of Experts in HCC-Design Clinical Trials: Design and endpoints of clinical trials in hepatocellular carcinoma. J Natl Cancer Inst 100: 698-711, 2008.

17. Asayama Y, Yoshimitsu K, Nishihara Y, Irie H, Aishima S, Taketomi A and Honda H: Arterial blood supply of hepatocellular carcinoma and histologic grading: Radiologic-pathologic correlation. AJR Am J Roentgenol 190: W28-W34, 2008.

18. Lee JH, Lee JM, Kim SJ, Baek JH, Yun SH, Kim KW, Han JK and Choi BI: Enhancement patterns of hepatocellular carcinomas on multiphasicmultidetector row CT: Comparison with pathological differentiation. Br J Radiol 85: e573-e583, 2012.

19. Gu KW, Kim YK, Min JH, Ha SY and Jeong WK: Imaging features of hepatic sarcomatous carcinoma on computed tomography and gadoxetic acid-enhanced magnetic resonance imaging. Abdom Radiol (NY) 42: 1424-1433, 2017.

20. Oishi K, Itamoto T, Amano H, Fukuda S, Ohdan H, Tashiro H, Shimamoto F and Asahara T: Clinicopathologic features of poorly differentiated hepatocellular carcinoma. J Surg Oncol 95: 311-316, 2007

21. Lu J, Xiong XZ, Li FY, Ye H, Lin YX, Zhou RX, Cai YL, Jin YW and Cheng NS: Prognostic significance of sarcomatous change in patients with hepatocellular carcinoma after surgical resection. Ann Surg Oncol 22 (Suppl 3): S1048-S1056, 2015.

22. Kawamura Y, Ikeda K, Hirakawa M, Yatsuji H, Sezaki H, Hosaka T, Akuta N, Kobayashi M, Saitoh S, Suzuki F, et al: New classification of dynamic computed tomography images predictive of malignant characteristics of hepatocellular carcinoma. Hepatol Res 40: 1006-1014, 2010.

23. Calderaro J, Couchy G, Imbeaud S, Amaddeo G, Letouzé E, Blanc JF, Laurent C, Hajji Y, Azoulay D, Bioulac-Sage P, et al: Histological subtypes of hepatocellular carcinoma are related to gene mutations and molecular tumour classification. J Hepatol 67: 727-738, 2017
24. Calderaro J, Ziol M, Paradis V and Zucman-Rossi J: Molecular and histological correlations in liver cancer. J Hepatol 71: 616-630, 2019.

25. Rodríguez-Hernández MA, Chapresto-Garzón R, Cadenas M, Navarro-Villarán E, Negrete M, Gómez-Bravo MA, Victor VM, Padillo FJ and Muntané J: Differential effectiveness of tyrosine kinase inhibitors in 2D/3D culture according to cell differentiation, p53 status and mitochondrial respiration in liver cancer cells. Cell Death Dis 11: 339, 2020.

26. Wang YY, Zhong JH, Su ZY, Huang JF, Lu SD, Xiang BD, Ma L, Qi LN, Ou BN and Li LQ: Albumin-bilirubin versus Child-Pugh score as a predictor of outcome after liver resection for hepatocellular carcinoma. Br J Surg 103: 725-734, 2016.

27. Oh IS, Sinn DH, Kang TW, Lee MW, Kang W, Gwak GY, Paik YH, Choi MS, Lee JH, Koh KC, et al: Liver function assessment using albumin-bilirubin grade for patients with very early-stage hepatocellular carcinoma treated with radiofrequency ablation. Dig Dis Sci 62: 3235-3242, 2017.

28. Lee IC, Hung YW, Liu CA, Lee RC, Su CW, Huo TI, Li CP, Chao Y, Lin HC, Hou MC, et al: A new ALBI-based model to predict survival after transarterial chemoembolization for BCLC stage B hepatocellular carcinoma. Liver Int 39: 1704-1712, 2019.

29. Ueshima K, Nishida N, Hagiwara S, Aoki T, Minami T, Chishina H, Takita M, Minami Y, Ida H, Takenaka M, et al: Impact of baseline ALBI grade on the outcomes of hepatocellular carcinoma patients treated with lenvatinib: A multicenter study. Cancers (Basel) 11: 952, 2019.

30. Hiraoka A, Kumada T, Atsukawa M, Hirooka M, Tsuji K, Ishikawa $\mathrm{T}$, Takaguchi $\mathrm{K}$, Kariyama $\mathrm{K}$, Itobayashi $\mathrm{E}$, Tajiri K, et al; Real-life Practice Experts for HCC (RELPEC) Study Group, HCC 48 Group (hepatocellular carcinoma experts from 48 clinics in Japan): Prognostic factor of lenvatinib for unresectable hepatocellular carcinoma in real-world conditions-Multicenter analysis. Cancer Med 8: 3719-3728, 2019.

31. Kudo M, Ueshima K, Ikeda M, Torimura T, Tanabe N, Aikata H, Izumi N, Yamasaki T, Nojiri S, Hino K, et al; TACTICS study group: Randomised, multicentre prospective trial of transarterial chemoembolisation (TACE) plus sorafenib as compared with TACE alone in patients with hepatocellular carcinoma: TACTICS trial. Gut 69: 1492-1501, 2020.

32. Jain RK: Normalization of tumor vasculature: An emerging concept in antiangiogenic therapy. Science 307: 58-62, 2005.

33. Shen H, Agarwal D, Qi R, Chalasani N, Liangpunsakul S, Lumeng L, Yoo $\mathrm{H}$ and Kwo P: Predictors of outcome in patients with unresectable hepatocellular carcinoma receiving transcatheter arterial chemoembolization. Aliment Pharmacol Ther 26: 393-400, 2007.

34. Sergio A, Cristofori C, Cardin R, Pivetta G, Ragazzi R, Baldan A, Girardi L, Cillo U, Burra P, Giacomin A, et al: Transcatheter arterial chemoembolization (TACE) in hepatocellular carcinoma (HCC): The role of angiogenesis and invasiveness. Am J Gastroenterol 103: 914-921, 2008.

35. Yang Y, Zhang Y, Iwamoto H, Hosaka K, Seki T, Andersson P, Lim S, Fischer C, Nakamura M, Abe M, et al: Discontinuation of anti-VEGF cancer therapy promotes metastasis through a liver revascularization mechanism. Nat Commun 7: 12680, 2016.

This work is licensed under a Creative Commons Attribution-NonCommercial-NoDerivatives 4.0 International (CC BY-NC-ND 4.0) License. 